\title{
Beiträge zur Kenntniss der untersalpetrigen Säure
}

\author{
(I. Mittheilung) \\ von
}

\section{Anton Thum,}

Assistent an der k. k. deutschen technischen Hochschule in Prag.

Aus dem chemisch-analytischen Laboratorium der k. k. deutschen technischen Hochschule in Prag.

(Vorgelegt in der Sitzung am 16. März 1893.)

Im Jahre 1863 entdeckte De Wilde ${ }^{1}$ die Thatsache, dass bei der Einwirkung von Natriumamalgam auf eine Lösung von Kalisalpeter nicht Wasserstoff frei wird, sondern ein Gasgemisch, das aus Stickstoff und Stickoxydul zu fast gleichen Theilen besteht. Als Fremy ${ }^{2}$ hierauf die Einwirkung von Natriumamalgam auf Nitrate untersuchte, fand er, dass zunächst Nitrite und bei weitergehender Reduction Hydroxylamin gebildet werden, während Stickstoff und Stickoxydul entweichen. Bei einem eingehenderen Studium dieser Reaction gelang es Maumené ${ }^{\mathbf{3}}$ einen Körper säureartiger Natur zu erhalten, welcher mit Silbernitrat ein Salz von der Formel $\mathrm{AgNO}$ liefern sollte, während der freien Säure die Formel $\mathrm{H}_{4} \mathrm{~N}_{2} \mathrm{O}_{3}$ zugeschrieben wurde. Divers ${ }^{4}$ bestätigte im Jahre 1871 die Angaben Maumené's und erhielt nach Einwirkung des Amalgams aus der alkalischen Lösung durch Neutralisation mit Essigsäure und Versetzen mit Silbernitrat einen schön

1 Bullet. de l'Académie Roy. de Belg. [2], 15, 560.

2 Compt. rend. 66, 1207.

3 Compt. rend. 70, 149.

4 London Roy. Soc. Proc. 19, 425. 
gelben Niederschlag, der sich als reines Salz einer neuen, der untersalpetrigen Säure erwies, welche sich aus dem Silbersalz durch Salzsäure frei machen liess. Van der Plaats ${ }^{1}$ studirte hierauf die Eigenschaften des Silberhyponitrits und der freien Hyponitrose, war aber nicht im Stande eine andere Darstellungsmethode ausfindig zu machen, noch ein anderes als das Silbersalz zu isoliren. Eine wesentliche Förderung erfuhr die Kenntniss dieser Stickstoffverbindungen durch die Arbeiten von Zorn ${ }^{2}$. Durch Einwirkung von Aethyljodid auf untersalpetrigsaures Silber erhielt dieser Forscher das Diazoäthoxan, das durch Ermittelung der Dampf́dichte für die untersalpetrige Säure die Formel $\mathrm{H}_{2} \mathrm{~N}_{2} \mathrm{O}_{2}$ wahrscheinlich machte. Eine weitere Bestätigung dieser Annahme war der Nachweis der Dibasicität mittelst der Existenz eines sauren Barytsalzes. Zorn fand auch, dass die Reduction von salpetrigsaurem Alkali mittelst Eisenhydroxydul sich zur Darstellung von untersalpetriger Säure eignet und dass sich solche bei der Elektrolyse von Natriumnitrit unter Anwendung von Quecksilberelektroden bildet. E. Mencke ${ }^{3}$ gibt an, durch Schmelzen von Natriumnitrat mit Eisenfeilspähnen untersalpetrigsaures Natrium erhalten zu haben, eine Beobachtung, welche sich nicht bestätigt hat. Wie Divers und Tamemasa $\mathrm{Haga}^{4}$ fanden, ist die Ausbeute bei der Reduction des Nitrits mittelst Eisenhydroxyduls eine ebenso geringe wie bei der Amalgammethode. Berthelot und Ogier ${ }^{5}$ sehen sich durch thermochemische Untersuchungen veranlasst für das Silbersalz die Formel $\mathrm{Ag}_{4} \mathrm{~N}_{4} \mathrm{O}_{5}$ anzunehmen, welche Annahme von Divers und Tame masa $\mathrm{Haga}$ wideregt wurde. Bei weiteren Versuchen ${ }^{6}$ fanden letztere Forscher, dass auch beim Einleiten von Stickoxyd in alkalische Zinnoxydullösung sich Alkalihyponitrit bildet, und nahmen dabei

1 D. Ges. Ber. 1877, 1507.

3 D. Ges. Ber. 1877, 1306; 1878, 1630; 1879, 1510; 1882, 1007 und 1258 .

3 Chem. Soc. J. 33, 401; Chem. News 37, 268.

4 Chem. Soc. J. 45, 78 .

3 Compt. rend. 96, 84; Bullet. Soc. chim. (2) 40, 401.

6 Chem. Soc. J. 47, 361 . 
intermediäre Nitrostannate an, welche, analog den Peiouze'schen ${ }^{1}$ Nitrosulfaten durch Akali erst in Hyponitrite umgewandelt werden. Wyndham R. Dunstan und T.S.Dymond ${ }^{2}$ haben die Einwirkung von Eisenhydroxydul gegen Nitrit genauer untersucht und gefunden, dass das Nitrit zuerst in Ammoniak, Stickstoff und Hyponitrit umgewandelt wird und dass letzteres durch weiteres Eisenhydroxydul zu Stickstoff reducirt werde; dabei soll intermediär ein $Z$ wischenproduct von der Formel $\mathrm{HN}(\mathrm{OH})_{z}$, also ein Dihydroxylamin, auftreten. Berthelot hat aus der Bildungswärme der Hyponitrose erklärlich gefunden, warum sich deren Anhydrid, das Stickoxydul, nicht direct mit den Alkalien zu Salzen verbindet. Die Kenntniss von den Salzen der untersalpetrigen Säure wurde von. Maquenne ${ }^{4}$ durch Darstellung der Hyponitrite und der Acetohyponitrite des Calciums und Strontiums bereichert.

\section{Über die Darstellungsmethoden der untersalpetrigen Säure.}

Von den bisher bekannten Bildungsweisen der untersalpetrigen Säure eignen sich nur zwei zur Darstellung derselben, nämlich jene der Reduction des salpetrigsauren Alkalis mittelst Natriums oder mittelst Eisenhydroxyduls. Nach Dunstan und Dymond geht die Reaction in folgenden Phasen vor sich:

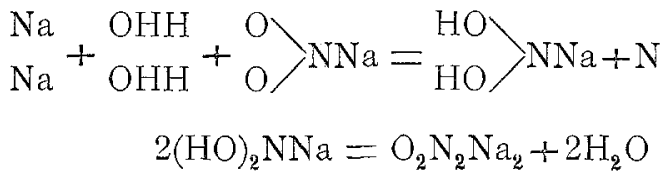

$$
\begin{aligned}
& (\mathrm{HO})_{2} \mathrm{NNa}+\mathrm{H}_{2}=\mathrm{H}_{2} \mathrm{NOH}+\mathrm{NaOH} \text {. }
\end{aligned}
$$

Durch Einwirkung überschüssigen Reductionsmittels soll aus dem Hydroxylamin Ammoniak und aus untersalpetriger Säure Stickstoff entstehen, wozu als Zerfallsproduct bereits gebildeter untersalpetriger Säure noch Stickoxydul kommt.

\footnotetext{
1 Chem. Soc. J. 47, 203.

2 Chem. Soc. J. 51, 648.

3 Compt. rend. $108,1286$.

4 Compt, rend. 108, 1303 .
} 
Bei der Reduction von Nitrit mittelst Natriumamalgam bekommt man kein Ammoniak und nur Spuren von Hydroxylamin, während bei derReduction mittelst Eisenoxydulhydrat massenhaft Ammoniak und keine Spur von Hydroxylamin auftritt.

Zur Ermittlung, ob bei Anwendung grösserer Natriummengen, als Divers vorschlägt, aus Nitrit nicht auch Ammoniak gebildet werden kann, wurde ein Versuch unter Kühlung gemacht, zu welchem die Mengenverhältnisse nach folgendem Schema gewählt waren:

$$
\mathrm{NaNO}_{2}+6 \mathrm{Na}+6 \mathrm{H}_{2} \mathrm{O}=\mathrm{NH}_{3}+7 \mathrm{NaOH}+\mathrm{H}_{2} \mathrm{O} .
$$

Durch den Geruch und durch das Nessler'sche Reagens konnte nach einigen Stunden deutlich Ammoniak nachgewiesen werden und war damit der früher hervorgehobene Widerspruch mit der Hypothese von Dunstan und Dymond beseitigt.

Von der Condensation zweier Moleküle Dihydroxylamin zu untersalpetriger Säure konnte vermuthet werden, dass eine solche durch den Alkaliüberschuss bei der Amalgammethode begünstigt wird. Ein directer Versuch bestätigte dies. Neutralisirt man das frei werdende Alkali durch einen heftigen Strom von Kohlensäure, so bildet sich keine untersalpetrige Säure und mehr Hydroxylamin.

Was das Auftreten des Stickstoffs anbelangt, so wird dasselbe einerseits mit der Einwirkung des Reductionsmittels auf bereits gebildete Hyponitrose erklärt, andererseits mit der Zersetzung von Hydroxylamin nach folgender Gleichung

$$
3 \mathrm{H}_{2} \mathrm{NOH}=\mathrm{N}_{2}+\mathrm{NH}_{3}+3 \mathrm{H}_{2} \mathrm{O} .
$$

Durch Untersuchungen von V. Meyer, ${ }^{1}$ von L. Crismer ${ }^{2}$ und von C. A. Lobry de Bruyn ${ }^{3}$ hat sich gezeigt, dass Hydroxylamin auch in alkalischer Lösung sich nicht leicht zersetzt, sondern ziemlich beständig ist; dann müsste nach

1 Liebig's Ann. 264, 116.

2 Bullet. Soc. chim. 6, 793.

3 Rec. trav. chim. X, 100. 
dieser Gleichung mit dem Stickstoff Ammoniak auftreten, was bei der Amalgammethode nicht der Fall ist. Betreffs der Bildung aus untersalpetriger Säure beweisen weiter unten angeführte Versuche, dass dieselbe durch Natriumamalgam nur wenig angegriffen wird. Die Stickstoffentwicklung kann daher nicht auf diese Ursachen zurückgeführt werden; hingegen ist es möglich, dass dieselbe entsteht durch Wechselwirkung von Körpern, aus welchen eine Abspaltung von Stickstoff gewärtigt werden kann. So können Dihydroxylamin und Hydroxylamin bei der labilen Natur beider nach folgender Gleichung reagiren:

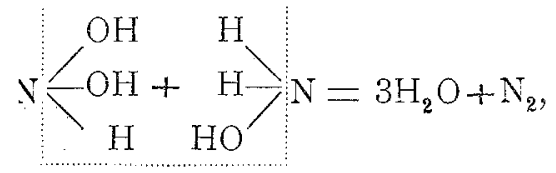

bevor eine völlige Condensation des ersteren zu untersalpetriger Säure und eine weitere Reduction des letzteren zu Ammoniak vor sich gehen konnte.

Das Auftreten des Stickoxyduls erklären Dunstan und Dymond als ein Resultat der Zersetzung concentrirter Hyponitritlösung durch Wasser: $\mathrm{Na}_{2} \mathrm{~N}_{2} \mathrm{O}_{2}+\mathrm{H}_{2} \mathrm{O}=2 \mathrm{NaOH}+\mathrm{N}_{2} \mathrm{O}$. $\mathrm{Da}$ man aber Lösungen von untersalpetriger Säure mit viel überschüssiger Natronlauge kochen kann, ohne nennenswerthe Gasentwicklung und Zerstörung der Säure zu bewirken, so ist es wahrscheinlich, dass ein Theil des Stickoxyduls seine Entstehung einer anderen Ursache verdankt. Diese Ansicht fand eine Bestätigung durch folgenden Versuch.

Eine verdünnte Nitritlösung wurde im langsamen Strom über Natriumamalgam fliessen gelassen; die Flüssigkeit passirte zweimal das mit der entsprechenden Amalgammenge beschickte Gefäss bis zur Zersetzung derselben. Der Versuch sollte in dieser Anordnung zugleich eine weitere Prüfung der Ansicht ermöglichen, nach welcher die Stickstoffentwicklung durch die Reduction bereits gebildeter Hyponitrose bedingt ist. Dies vorausgesetzt, müsste eine solche Anordnung eine Vermehrung der Ausbeute an Hyponitrit bewirken; dazu müsste eine weitere Vermehrung kommen wenn die Stickoxydul- 
entwicklung auf einen Zerfall von untersalpetriger Säure wegen hoher Concentration zurückzuführen ist. Die Ausbeute war wie bei vielen anderen Darstellungen nach Divers' Methode circa 7 Proc. der theoretischen. Van der Plaats bekam bei seinen Versuchen mit Kaliumnitrit 6.5 Proc. Ausbeute, Zorn gleichfalls 7 Proc. Die Ursache dieser geringen Ausbeute ist nach dem Vorangeführten jedenfalls in dem Umstande zu suchen, dass die Bildung von Hyponitrose aus dem Dihydroxylamin viel langsamer vor sich geht als die weitere Zersetzung und Reduction dieses unbeständigen Körpers.

Die Darstellung von Hyponitrit aus Nitrit mittelst Eisenoxydulhydrats hat vor der Amalgammethode keine Vortheile. Die Oxydation des Eisenoxyduls geht rasch bis zu Oxyduloxyd, dann nur sehr langsam weiter zu Oxyd. Die Ausbeuten sind etwas geringer als jene mittelst Amalgams und dies ist wieder bedingt durch die Trägheit, mit der Eisenoxydul auf Nitrit wirkt, während es die daraus hervorgegangenen Reductionsproducte weit leichter angreift. Die Hauptmasse des Stickstoffs tritt in elementarer, dann in Form von Stickoxydul und Ammoniak auf. Auch hier bietet die Annahme eines intermediär gebildeten Dihydroxylamins eine ungezwungene Erklärung für die Bildung aller bei dem Process auftretenden Producte. Die Tendenz dieses Körpers in Hyponitrose überzugehen ist hier geringer als bei der Amalgammethode, da kein freies Alkali anwesend ist. Ein Versuch die Reduction bei Gegenwart von freiem Aikali auszuführen bestätigte die Angaben von Dunkan und Dymond, dass in diesem Falle das Eisenoxydul unwirksam ist. Es ist nicht Wunder zu nehmen, dass bei dieser Methode niemals Hydroxylamin auftritt, indem dieses bei der trägen Einwirkung des Eisenoxyduls stets einen Überschuss des Reductionsmittels vorindet und sofort in Ammoniak überführt wird.

\section{Bildung von Hyponitrose aus Hydroxylamin.}

V. Meyer ${ }^{1}$ hat gelegentlich seiner Versuche über Nitrolsäuren gezeigt, dass concentrirte Lösungen von Hydroxylamin-

1 Ann. Pharm. Chem. 175, 141. 
salz und Natriumnitrit im Sinne der folgenden Gleichung auf einander wirken:

$$
\mathrm{H}_{3} \mathrm{NO}+\mathrm{HNO}_{2}=2 \mathrm{H}_{2} \mathrm{O}+\mathrm{N}_{2} \mathrm{O}
$$

Es lag nahe zu erwarten, dass auf diesem Wege die Bildung von Hyponitrose möglich sei, wenn blos ein Molekül Wasser austritt. Bei der Auffasung der untersalpetrigen Säure als Azokörper konnte die Bildung nach folgendem Process vor sich gehen:

$$
\mathrm{HON}\left|\mathrm{H}_{2}+\mathrm{O}\right| \mathrm{NOH}=\mathrm{H}_{2} \mathrm{O}+\mathrm{HON}=\mathrm{NOH}
$$

Dann war anzunehmen, dass analog der Bildung der Diazokörper der aromatischen Reihe auch die Bildung dieses Körpers aus seinem Amin (dem Hydroxylamin) und salpetriger Säure besonders bei niederer Temperatur stattfinden werde. In der That lehrten die darauf zielenden Versuche, dass auf diese Weise eine wenn auch untergeordnete Bildung von Hyponitrose stattfindet. Lässt man nach dieser Gleichung berechnete Mengen von salzsaurem Hydroxylamin und Natriumnitrit auf einander wirken, so erhält man, wenn die heftige Stickoxydulentwicklung nachgelassen hat, durch Fällen mit Silbernitrat einen blassgelben Niederschlag, der beim Behandeln mit Salpetersäure ungelöstes Chlorsilber zurücklässt, während Silberhyponitrit in Lösung geht, das, mit Ammoniak wieder ausgefällt, bei der Analyse folgende Zahlen ergab: $0.5318 \mathrm{~g}$ im Vacuum getrocknetes Silbersalz lieferten $0.5438 \mathrm{~g} \mathrm{AgCl}$, enthielten also $77 \% \mathrm{Ag}$. Reines Silberhyponitrit verlangt $78 \cdot 26 \%$ Ag. Durch Anwendung von schwefelsaurem Hydroxylamin vermeidet man die mitVerlust verbundene Trennung vom Chlorsilber. Die Ausbeute betrug etwas über $2 \%$ der theoretischen, die Hauptmasse des Stickstoffs aus beiden Bestandtheilen entweicht also in Form von Stickoxydul. Bei Anwendung alkalischer Lösungen zeigt sich keine Gasentwicklung und keine Bildung von Hyponitrit. Auch in essigsaurer Lösung und bei Zusatz von Calciumcarbonat zur Neutralisation der entstehenden freien Säure tritt keine Bildung ein. Aus diesen Versuchen geht hervor, dass die saure Reaction, welche anfangs nur durch die Lösung des Hydroxylaminsalzes bewirkt wird und die sich während des Processes 
verstärkt, zur Bildung von Stickoxydul und untersalpetriger Säure nothwendig ist. Auch bei Anwendung von Druck zeigte sich keine Vermehrung der Ausbeute.

Um die Reaction systematisch studiren zu können, strebte ich eine Methode zu finden, welche ein quantitatives Verfolgen der dabei auftretenden Processe ermöglichte. Versuche, die ich darüber mit Kaliumpermanganat angestellt habe, führten zu Resultaten, die ziemlich befriedigend sind. Wie bekannt lässt sich salpetrige Säure in saurer Lösung mit Permanganat genau titriren. Nach Untersuchungen von E. Donath ${ }^{1}$ widersteht salpetrige Säure in alkalischer Lösung der Einwirkung von Chamäleonlösung. Nun hat L. Storch" gelegentlich einer Arbeit, deren Resultate derselbe demnächst veröffentlichen will, festgestellt, dass sich bei Anwendung von arseniger Säure als Reductionsmittel Permanganat auch in alkalischer Lösung glatt titriren lässt, eine Beobachtung, deren Richtigkeit ich auf Grund meiner Versuche bestätigen kann. Da, wie ich zeigen werde, untersalpetrige Säure und ebenso Hydroxylamin in alkalischer Lösung durch Permanganat angegriffen werden und erstere glatt zu salpetriger Säure, letzteres zu einem bestimmten intermediären Oxydationsproduct oxydirt werden kann, so war ein Weg gefunden um durch parallelgehende Titration in alkalischer und andererseits in saurer Lösung mit Kaliumpermanganat alle bei diesen Reactionen auftretenden Stickstoffsauerstoffverbindungen, mit.Ausnahme des Stickoxyduls, quantitativ zu bestimmen und den Gang des Processes quantitativ zu verfolgen, zumal die Bestimmung des Hydroxylamins leicht durch schon bekannte massanalytische Methoden erfolgen kann. Die vollständige Oxydation des Hydroxylamins in saurer Lösung bis zu Salpetersäure bietet gewisse Schwierigkeiten und bin ich noch damit beschäftigt die Bedingungen zu ermitteln, unter welchen diese Oxydation quantitativ vor sịch geht.

Die analytischen Belege für die Bestimmbarkeit der Hyponitrose mittelst Permanganats sowohl in saurer als auch in

1 Monatsh. f. Chem. 1890, 15.

2 Privatmittheilung. 
alkalischer Lösung werden weiter unten angeführt, jene über das Verhalten der salpetrigen Säure und des Hydroxylamins lasse ich hier folgen:

Eine Permanganatlösung ergab bei der Titration. mit Oxalsäure einen Gehalt von $3 \cdot 3228 g \mathrm{KMnO}_{4}$ in 1 l. Diese wurde nun mit arseniger Säure $\left(3.0094 \mathrm{~g} \mathrm{As}_{2} \mathrm{O}_{3}\right.$ in $\left.1 l\right)$ titrirt, deren Lösung unter Zusatz von etwas Natronlauge bewerkstelligt war. Die Titration wird in heisser, alkalischer Lösung ausgeführt und geht, wie L. Storch (1. c.) nachgewiesen hat, genau nach folgender Gleichung vor sich:

$$
3 \mathrm{As}_{2} \mathrm{O}_{3}+2 \mathrm{Mn}_{2} \mathrm{O}_{7}=3 \mathrm{As}_{2} \mathrm{O}_{5}+4 \mathrm{MnO}_{2} \text {. }
$$

Man setzt so lange arsenige Säure zu, bis die über dem braunen Niederschlag stehende Flüssigkeit keinen Stich ins Grüne mehr hat. Bei den angestellten Versuchen wurden folgende Zahlen erhalten:

$\begin{array}{lll}\underbrace{\mathrm{K} \mathrm{MnO}_{4}}_{20 \mathrm{~cm}^{3}} & \underbrace{\text { Natronlauge (norm.) }}_{6 \mathrm{~cm}^{3}} & \underbrace{\mathrm{As}_{2} \mathrm{O}_{3}}_{10} \\ 24 \cdot 1 & 3 & 10 \cdot 4 \mathrm{~cm}^{3} \\ 24 \cdot 1 & 6 & 24 \cdot 5\end{array}$

Daraus rechnet sich für das Permanganat ein Gehalt von $3 \cdot 264 \mathrm{~g} \mathrm{KMnO}_{4}$ in $1 l$, welcher Werth mit dem früheren ziemlich stimmt.

Eine andere Lösung von Kaliumpermanganat, welche zu allen folgenden Titrationen benützt wurde, ergab bei der Titerstellung mit Oxalsäure einen Gehalt von $3 \cdot 24447 g$, bei der Titration mit arseniger Säure einen Gehalt von $3 \cdot 2882 \mathrm{~g}$ $\mathrm{KMnO}_{4}$ in 1 l. Die kleine Erhöhung des letzteren Werthes ist, wie ich mich überzeugt habe, durch Verunreinigungen der Natronlauge bedingt.

Die Titration des Hydroxylamins wurde in der Weise ausgeführt, dass zu einem Überschuss von alkalischer Permanganatlösung die Hydroxylaminlösung hinzufliessen gelassen wurde. Nach Einwirkung während einer Stunde in der Kälte wurde zum Kochen erhitzt und mit arseniger Säure zurücktitrirt. Wie aus den folgenden Versuchen ersichtlich ist, erscheint es nothwendig, zur völligen Oxydation einen ziemlich 
grossen Überschuss von Permanganat anzuwenden; die erhaltenen Resultate sind dann constant. In den folgenden Versuchen wurden je $10 \mathrm{~cm}^{3}$ der schwefelsauren Hydroxylaminlösung $(5 \cdot 1 \mathrm{~g}$ Sulfat in $1 \mathrm{l})$ unter verschiedenen Bedingungen titrirt.

\begin{tabular}{|c|c|c|c|c|}
\hline$\underbrace{\mathrm{KMnO}_{4}}$ & Natronl. n. & Hydroxylamin & $\underbrace{\mathrm{As}_{2} \mathrm{O}_{3}}$ & $\begin{array}{l}\text { Permanganat- } \\
\text { verbrauch }\end{array}$ \\
\hline $30 \mathrm{~cm}^{3}$ & $20 \mathrm{~cm}^{3}$ & $10 \mathrm{~cm}^{3}$ & $1 \cdot 4 \mathrm{~cm}^{3}$ & $28.6 \mathrm{~cm}^{3}$ \\
\hline 41 & 20 & 10 & $11 \cdot 7$ & $29 \cdot 6$ \\
\hline 40 & 20 & 10 & $10 \cdot 7$ & $29 \cdot 6$ \\
\hline 50 & 10 & 10 & $19 \cdot 9$ & $30 \cdot 1$ \\
\hline 50 & 10 & 10 & $19 \cdot 9$ & $30 \cdot 1$ \\
\hline
\end{tabular}

Berechnet man für die gewählten Concentrationsverhältnisse die Menge Permanganatlösung, welche nothwendig wäre, die angewandte Hydroxylaminmenge zu Hyponitrose oder zu Nitrose zu oxydiren, so beträgt dieselbe

$$
\frac{\text { für Hyponitrose }}{20 \cdot 2 \mathrm{~cm}^{3}} \quad \frac{\text { für salpetrige Säure }}{40 \cdot 4 \mathrm{~cm}^{3}}
$$

Die gefundene Zahl von $30.1 \mathrm{~cm}^{3}$ Permanganat weist also unzweifelhaft darauf hin, dass sich ein Körper bildet, der in seinem Sauerstoffgehalte genau zwischen den obengenannten beiden Körpern steht.

Es liegt nahe, anzunehmen, dass ein solches Oxydationsproduct, das durch Anlagerung von einem Sauerstoffatom an das Molekül der untersalpetrigen Säure entstanden sein muss und möglicher Weise unter Auflösung der Doppelbindung der beiden Stickstoffatome gebildet zu denken wäre, sohin einer Formel $\mathrm{O} \mathrm{N}_{\mathrm{N}-\mathrm{OH}}^{\mathrm{N}-\mathrm{OH}}$ entspricht. Diese Formel stünde im Einklange mit der Beziehung der Azoxykörper zu den Azokörpern, in deren Reihe die Hyponitrose einbezogen werden muss, und es schien mir daher passend, dieses vermuthlich intermediäre Oxydationsproduct als Azoxykörper aufzufassen und folgerichtig als Azohydroxyl zu bezeichnen. Wir hätten dann folgende Verbindungen, deren genetischer Zusammenhang unter einander und deren Analogie mit den entsprechenden 
aromatischen Verbindungen ohne jeden Commentar in die Augen fällt.

$\underbrace{\text { Ammoniak }}_{\mathrm{H}_{2} \mathrm{NH}} \quad \underbrace{\text { Hydroxylamin }}_{\mathrm{H}_{2} \mathrm{NOH}}$

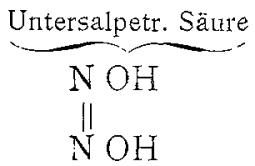

$\underbrace{\text { Azoxykörper }}_{\substack{\mathrm{N} \mathrm{OH} \\ \mathrm{N}-\mathrm{OH}}}$

Als Product einer noch weiter gehenden Oxydation erscheint dann salpetrige Säure, welche sich in der That aus dem Azokörper, der Hyponitrose, unter Zerreissung der Stickstoffbindung durch Oxydation mit Permanganat in alkalischer Lösung bildet, wie später gezeigt werden wird. Der Azoxykörper ist aber, wie die obigen Versuche zeigen, gegen Permanganat in alkalischer Lösung beständig und die Oxydadation von Hydroxylamin durch Chamäleon geht bloss bis zu diesem Körper. Es muss weiteren Untersuchungen, deren Ziel es sein wird, diese vermeintlich neue Sauerstoffverbindung des Stickstoffs eventuell zu isoliren und ihre Eigenschaften genauer zu studiren, vorbehalten bleiben zu entscheiden, ob diese Auffassung begründet erscheint, und beabsichtige ich bei Vornahme dieser Arbeiten, die ich mir vorbehalte, auch die eventuelle Existenz eines Hydrazokörpers, der als Mittelglied zwischen Hydroxylamin und untersalpetriger Säure noch fehlt, ins Auge zu fassen, denn es drängt sich die Vermuthung auf, dass zwischen dem Hydroxylamin und der salpetrigen Säure folgende Reihe von Körpern liegt.

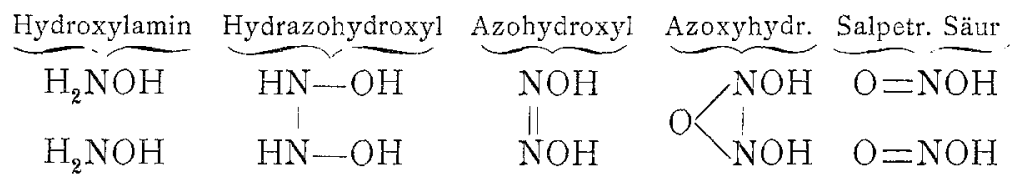

Weitere Versuche der Titration von Hydroxylamin in anderen Concentrationen mit Kaliumpermanganat lieferten Resultate, welche mit den früher erhaltenen und mit dieser Theorie sehr gut übereinstimmen und lasse ich diese der Vollständigkeit halber folgen:

Hydroxylaminlösung $(1 \cdot 263 \mathrm{~g}$ Sulfat in $1 \mathrm{l})$

Permanganatlösung $\left(3 \cdot 24447 \mathrm{~g} \mathrm{KMnO}_{4}\right.$ in $\left.1 l\right)$

Arsenige Säure $\left(3 \cdot 0094 \mathrm{~g} \mathrm{As}_{2} \mathrm{O}_{3}\right.$ in $\left.1 l\right)$ 


\begin{tabular}{|c|c|c|c|c|}
\hline$\underbrace{\mathrm{KMnO}_{4}}$ & $\begin{array}{c}\begin{array}{c}\text { Natronlauge } \\
\text { (norm.) }\end{array} \\
\end{array}$ & $\underbrace{\text { Hydroxyl. }}$ & $\underbrace{\mathrm{As}_{2} \mathrm{O}_{3}}$ & $\begin{array}{l}\begin{array}{l}\text { Permangan- } \\
\text { verbrauch }\end{array} \\
.\end{array}$ \\
\hline $50 \mathrm{~cm}^{3}$ & $20 \mathrm{~cm}^{3}$ & $10 \mathrm{~cm}^{3}$ & $43 \cdot 95 \mathrm{~cm}^{3}$ & $7 \cdot 25 \mathrm{~cm}^{3}$ \\
\hline 40 & 40 & 20 & $25 \cdot 8$ & $14 \cdot 9$ \\
\hline 40 & 40 & 20 & $25 \cdot 75$ & $14 \cdot 9$ \\
\hline
\end{tabular}

Für $10 c c$ Hydroxylaminlösung berechnet sich bej Oxydation bis zu Azohydroxyl ein Verbrauch von $7 \cdot 5 \mathrm{~cm}^{3}$

Hydroxylaminlösung ( $1.0772 \mathrm{~g}$ Chlorid in $1 \mathrm{l}$ )

Permalıganat- und Arsenigsäurelösung wie oben.

\begin{tabular}{|c|c|c|c|c|}
\hline$\underbrace{\mathrm{KMnO}_{4}}$ & $\begin{array}{c}\begin{array}{c}\text { Natronlauge } \\
\text { (norm.) }\end{array} \\
\end{array}$ & Hydroxyl. & $\underbrace{\mathrm{As}_{2} \mathrm{O}_{3}}$ & $\begin{array}{r}\text { Permangan } \\
\text { verbrauch }\end{array}$ \\
\hline $41 \mathrm{~cm}^{3}$ & $40 \mathrm{~cm}^{3}$ & $20 \mathrm{~cm}^{3}$ & $25 \cdot 7 \mathrm{~cm}^{3}$ & $16 \mathrm{~cm}^{3}$ \\
\hline 40 & 40 & 20 & $24 \cdot 6$ & 16 \\
\hline
\end{tabular}

Daraus ergibt sich für $10 \mathrm{~cm}^{3}$ Hydroxylaminlösung ein Verbrauch von $8 \mathrm{~cm}^{3}$ gegen $7 \cdot 5 \mathrm{~cm}^{3}$ theoretisch. Diese Abweichung ist bedingt durch geringe Verunreinigungen der angewandten Hydroxylaminsalze, kann jedoch ihrer Geringfügigkeit halber wohl vernachlässigt werden.

Hydroxylamin reagirt heftig mit einigen Metalloxyden z. B. mit Quecksilberoxyd, Silberoxyd, Kupferoxyd; dabei findet in untergeordnetem Masse auch Bildung von Hyponitrose statt. Lässt man Quecksilberoxyd als Brei allmählich zu der geküh1ten, alkalisch gemachten Lösung von Hydroxylamin hinzufliessen, so vollzieht sich partiell folgender Process:

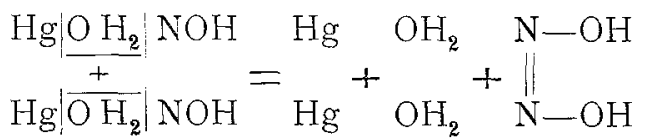

Neben Stickoxydul und untersalpetriger Säure wird stets auch etwas salpetrige Säure gebildet.

Von besonderem Interesse ist die schon angedeutete Bildung von Hyponitrose aus Hydroxylamin durch alkalisches Kupferoxyd, da die gewöhnliche Methode des Nachweises von Hydroxylamin auf Anwendung dieses Reagens sich gründet. Das alkalische, von Hydroxylamin freie Filtrat gibt, mit Silbernitrat versetzt, einen Niederschlag, der nach dem Lösen in verdünnter Schwefelsäure beim Überschichten mit verdünntem 
Ammoniak die gelbe Hyponitritzone liefert. Die Ausbeuten an Hyponitrit betragen jedoch nur 3 Proc. der Menge, welche nach folgendem Schema zu erwarten wäre:

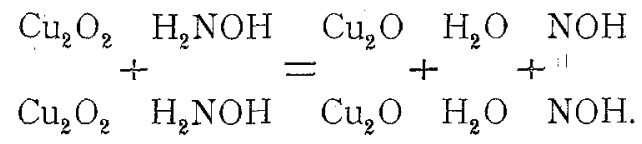

Auch bei dieser Reaction ist man im Stande die Bildung von Spuren salpetriger Säure nachzuweisen. Während bei Kochhitze, wie Donath ${ }^{1}$ und Meyeringh ${ }^{2}$ nachgewiesen haben, das Kupferoxyd den ganzen Stickstoff des Hydroxylamins in Stickoxydul verwandelt, findet also bei Kühlung eine untergeordnete Bildung von untersalpetriger und salpetriger Säure statt.

Die Bildung von Hyponitrose aus Hydroxylamin wird auch durch andere Oxydationsmittel wie Wasserstoffsuperoxyd, alkalische Ferricyankaliumlösung bewirkt, doch immer nur in geringer Menge. Die Hauptmasse des Stickstoffs tritt entweder in Form von Stickoxydul oder von salpetriger Säure auf, je nach der angewandten Menge des Oxydationsmittels. In wie weit auch durch diese Oxydationsmittel die Bildung von Azoxyhydroxyl stattfindet und inwieweit auch durch Kaliumpermanganat aus Hydroxylamin unter gewissen Umständen sich Hyponitrose bildet, müssen weitere Versuche lehren. Da die Untersuchung über diesen Gegenstand noch nicht abgeschlossen ist, so begnüge ich mich, auf diese vorläufigen Ergebnisse hinzuweisen.

\section{Eigenschaften der untersalpetrigen Säure.}

Bei Versuchen aus trockenem Silberhyponitrit und trockenem Schwefelwasserstoff wasserfreie untersalpetrige Säure darzustellen, zeigte sich bald, dass diese selbst bei niederen Temperaturen sehr unbeständig ist und sich leicht explosionsartig zersetzt. Die Eigenschaften wurden deshalb an der Auflösung in Wasser studirt, welche wie bekannt aus dem Silber-

1 Ber. d. Ges. 1877, 766.

2 Das. p. 1940. 
salz durch Salzsäure erhalten wird. Die Lösung stellt eine farblose, stark sauer reagirende Flüssigkeit dar, die selbst beim Kochen gegen verdünnte Säuren und Alkalien beständig ist. Durch Kaliumpermanganat tritt sowohl in saurer als auch in alkalischer Lösung Oxydation ein, die quantitativ verläuft.

In einer frisch bereiteten Lösung von untersalpetriger Säure wurde der Gehalt mittelst des Silbersalzes gewichtsanalytisch unter genauem Neutralisiren mit Ammoniak bestimmt. Das Silberhyponitrit aus $100 c c$ Lösung lieferte beim Glühen $0.1968 \mathrm{~g}$ Silber; die Lösung enthielt demnach $0.05649 \mathrm{~g}$ $\mathrm{H}_{2} \mathrm{~N}_{2} \mathrm{O}_{2}$ in $100 \mathrm{~cm}^{3}$. Dieselbe Lösung wurde nun ziemlich gleichzeitig mit Chamäleon in saurer Lösung titrirt. $\mathrm{Zu} 50 \mathrm{~cm}^{3}$ Permanganat $\left(3 \cdot 24447 \mathrm{~g} \mathrm{KMnO}_{4}\right.$ in $\left.1 l\right)$ wurden $20 \mathrm{cc}$ Hyponitrose zugefügt, eine Viertelstunde bei gewöhnlicher Temperatur einwirken gelassen, dann mit verdünnter Schwefelsäure angesäuert und nach nochmals viertelstündiger Einwirkung zurücktitrirt. Dabei war, übereinstimmend in mehreren Versuchen, der Permanganverbrauch $14.1 \mathrm{~cm}^{3}$, was einem Gehalt von $0.0561 \mathrm{~g} \mathrm{H}_{2} \mathrm{~N}_{2} \mathrm{O}_{2}$ entspricht, wenn man der Rechnung $\mathrm{zu}$ Grunde legt, dass die Oxydation bis zu Salpetersäure geht, wie auch die qualitative Prüfung erweist. Die Reaction verläuft also, bei Einhaltung der gewählten Verhältnisse, nach folgendem Schema:

$$
\begin{gathered}
5 \mathrm{H}_{2} \mathrm{~N}_{2} \mathrm{O}_{2}+8 \mathrm{KMNO}_{4}+12 \mathrm{H}_{2} \mathrm{SO}_{4}= \\
=10 \mathrm{HNO}_{3}+4 \mathrm{~K}_{2} \mathrm{SO}_{4}+8 \mathrm{MnSO}_{4}+12 \mathrm{H}_{2} \mathrm{O}
\end{gathered}
$$

Ein Überschuss von Schwefelsäure ist ohne Einfluss auf die Reaction, während ein Mangel dieselbe beeinträchtigt.

Die wässerige Lösung von untersalpetriger Säure zersetzt sich allmählich in Stickoxydul und Wasser. Die obige Bestimmungsmethode gestattet diesen Zerfall quantitativ zu verfolgen. Derselbe wird durch wenig Alkali wesentlich begünstigt, während viel Alkali verzögernd einzuwirken scheint.

Untersalpetrige Säure lässt sich mit Permanganat auch in alkalischer Lösung titriren; die Oxydation geht dabei bis $\mathrm{zu}$ salpetriger Säure. Eine Lösung von Hyponitrose ergab bei der Permanganattitration in saurer Lösung einen Gehalt von 
$0.97485 \mathrm{~g} \mathrm{H}_{2} \mathrm{~N}_{2} \mathrm{O}_{2}$ in 1 l. Die Titration in alkalischer Lösung ergab folgende Zahlen:

\begin{tabular}{|c|c|c|c|c|c|}
\hline & $\underbrace{\mathrm{KMnO}_{4}}$ & Natronl. (n.) & $\underbrace{\mathrm{H}_{2} \mathrm{~N}_{2} \mathrm{O}_{2}}$ & $\underbrace{\mathrm{As}_{2} \mathrm{O}_{3}}$ & $\begin{array}{l}\text { Permangan } \\
\text { verbrauch }\end{array}$ \\
\hline (heiss) & $50 \mathrm{~cm}^{3}$ & $20 \mathrm{~cm}^{3}$ & $10 \mathrm{~cm}^{3}$ & $40 \cdot 5 \mathrm{~cm}^{3}$ & $10 \cdot 6 \mathrm{~cm}^{3}$ \\
\hline (heiss) & 50 & 20 & 10 & $40 \cdot 5$ & $10 \cdot 6$ \\
\hline (kalt) & 30 & 20 & 10 & $21 \cdot 6$ & $9 \cdot 6$ \\
\hline
\end{tabular}

Die Concentration der Permanganatlösung und der arsenigen Säure war wie früher angegeben. Aus obigem Gehalte der Hyponitroselösung rechnet sich ein Permanganatverbrauch von $10 \cdot 3 \mathrm{~m}^{3}$. Die thatsächlich gefundene Zahl ist also etwas zu hoch; wie ich zeigen werde, ist dies darauf zurückzuführen, dass durch die Einwirkung des Permanganatüberschusses in der Hitze eine wenn auch geringe Oxydation der zuerst gebildeten salpetrigen Säure zu Salpetersäure stattfindet.

Folgende Blankversuche machen den Einfluss der Zeitdauer ersichtlich.

\begin{tabular}{|c|c|c|c|c|c|}
\hline$\underbrace{\mathrm{KMnO}_{4}}$ & Natroni. (n.) & $\begin{array}{l}\mathrm{NaNO}_{2} \\
(1 / 10 \text { n. })\end{array}$ & $\underbrace{\mathrm{As}_{2} \mathrm{O}_{3}}$ & $\begin{array}{l}\text { Kochen } \\
\text { während }\end{array}$ & $\begin{array}{c}\begin{array}{c}\text { Permangan- } \\
\text { verbrauch }\end{array} \\
\text {. }\end{array}$ \\
\hline $20 \mathrm{~cm}^{3}$ & $20 \mathrm{~cm}^{3}$ & $20 \mathrm{~cm}^{3}$ & $19 \cdot 5 \mathrm{~cm}^{3}$ & $1 / 2 \mathrm{St}$. & $1.0 \mathrm{~cm}^{3}$ \\
\hline 20 & 20 & 20 & $18 \cdot 2$ & 1 & $2 \cdot 3$ \\
\hline 20 & 20 & 20 & $17 \cdot 8$ & 2 & $2 \cdot 7$ \\
\hline
\end{tabular}

Die Oxydation der untersalpetrigen Säure mit Kaliumpermanganat geht also in saurer Lösung zu Salpetersäure, in alkalischer Lösung zu salpetriger Säure vor sich.

Salpetrige Säure wirkt auf untersalpetrige Säure zersetzend ein. Lässt man sehr verdünnte Lösungen beider Säuren auf einander wirken, so zeigt sich bald Gasentwicklung und nach wenigen Minuten ist die Hyponitrose verschwunden, wenn genügend salpetrige Säure vorhanden war. Quantitativ angestellte Versuche machen es wahrscheinlich, dass ein Molekül untersalpetrige Säure $\left(\mathrm{H}_{2} \mathrm{~N}_{2} \mathrm{O}_{2}\right)$ und ein Molekül Nitrose $\left(\mathrm{HNO}_{2}\right)$ mit einander in Wechselwirkung treten. Die Untersuchung der Reaction auf titrimetrischem Wege mit Hilfe der angegebenen Methoden dürfte Aufklärung darüber verschaffen, ob diese Vermuthung richtig ist. 
Wie Zorn (1. c.) durch den Nachweis der Existenz eines sauren Barytsalzes dargethan hat, ist die untersalpetrige Säure zweibasisch. Ein weiterer Beweis dafür liegt in dem Verhalten derselben gegen Alkalien bei Anwendung von Phenolphtaleïn als Indicator. In dem Momente als alle Säure in das saure Salz verwandelt ist, wird die Bildung von neutralem Salz durch die Rothfärbung angezeigt. Zur Anwendung kam eine Lösung von $0.97485 \mathrm{~g} \mathrm{H}_{2} \mathrm{~N}_{2} \mathrm{O}_{2}$ in $1 l$ und eine Kalilauge, von der $1 \mathrm{~cm}^{3}$ $0.02553 \mathrm{~g} \mathrm{KOH}$ enthielt. $100 \mathrm{~cm}^{3}$ der Säure brauchten, übereinstimmend in zwei Versuchen, $3.5 \mathrm{~cm}^{3}$ Kalilauge. Das ergibt einen Kalihydratverbrauch von $0.088355 \mathrm{~g}$. Bei Absättigung bis zu saurem Salz rechnet sich aus dem obigen Gehalte ein Verbrauch von $0.08805 \mathrm{~g} \mathrm{KOH}$. Beide Zahlen stimmen so gut, dass daraus die Möglichkeit einer directen acidimetrischen Gehaltsbestimmung unter Anwendung von Phenolphtaleïn gefolgert werden kann. Lackmus verhält sich ähnlich wie Phenolphtaleïn; auf Methylorange wirkt untersalpetrige Säure nicht ein. Aus Carbonaten und Bicarbonaten treibt Hyponitrose keine Kohlensäure aus; doch auch umgekehrt werden Hyponitrite von Kohlensäure nicht zersetzt.

Über das Verhalten der untersalpetrigen Säure gegenüber Jodkalium in saurer Lösung widersprechen sich die Angaben von Divers und Van der Plaats. Ersterer führt an; dass freie untersalpetrige Säure Jodlösung entfärbt und die Stärkebläuung verhindert, während Van der Plaats findet, dass Jodkaliumstärke dadurch blau gefärbt wird.

Diese Angabe fand ich ebensowenig bestätigt, wie jene von Divers.

Eine frisch bereitete, völlig reine Lösung von Hyponitrose färbt (mit Schwefelsäure) angesäuerte Jodkaliumstärkelösung nicht blau und scheidet kein Jod aus. Eine Verhinderung der mittelst salpetriger Säure erzeugten Blaufärbung war auch nicht bemerkbar. Um zu constatiren, ob eine Jodabsorption stattfindet, wurde folgender Versuch gemacht. Untersalpetrige Säure wurde mit Jodlösung geschüttelt, das überschüssige Jod durch Schwefelkohlenstoff entzogen und die so behandelte Hyponitroselösung mit Zink und Schwefelsäure und 
mittelst Natriumamalgam reducirt. In beiden Fällen liess sich keine Spur von aufgenommenem Jod constatiren.

Hyponitrose macht also aus Jodkalium in saurer Lösung weder Jod frei, noch hindert sie die Blaufärbung der Jodstärke. Eine scheinbare Jodabsorption und Verhinderung der Blaufärbung kann gefunden werden, wenn die Hyponitroselösung saures Silberhyponitrit enthält, durch welches Jodsilber gebildet wird. Bei Anwendung von viel Hyponitrose und wenig salpetriger Säure kann es auch scheinen, dass die Blaufärbung gehindert wird, da beide Körper sich, wie früher erwähnt, zerstören.

Gegen reducirende Agentien erweist sich die untersalpetrige Säure sehr beständig. Bei einem Versuch wurde Hyponitrose in verdünnter Lösung mit einem Überschuss von Zink und Schwefelsäure gekocht und durch hierauf folgende 6stündige Einwirkung bei gewöhnlicher Temperatur nicht zum Verschwinden gebracht. Auch derReduction in alkalischerLösung widersteht der Körper. Bei einem diesbezüglich angestellten Versuche wurde eine bestimmte Menge untersalpetriger Säure durch Behandlung mit mehr als der doppelten Menge Natriumamalgam als zur Überführung des Gesammtstickstoffes in Ammoniak nöthig war, nicht völlig zerstört.

Versetzt man eine Hyponitroselösung mit so viel Alkali, als zur Bildung von saurem Alkalisalz nöthig ist, so erhält man mit einer solchen Lösung bei Einwirkung auf viele Metallsalze Niederschläge, welche sich als die betreffenden Salze der untersalpetrigen Säure erweisen. Über deren Analyse und Eigenschaften will ich in einer späteren Mitheilung berichten.

An dieser Stelle sei es mir gestattet, Herrn Professor. Dr. Gintl für die mannigfache Förderung, welche derselbe mir bei dieser Arbeit zu Theil werden liess, meinen besten Dank auszusprechen. Auch Herrn Docenten L. Storch bin ich für viele werthvolle Rathschläge zu Dank verpflichtet. 